\title{
Cáncer de Mama, siete años de experiencia quirúrgica en el Hospital Oncológico "Julio Enrique Paredes C".
}

\section{*Correspondencia:}

alexandra.gavilanes@gmx.es

Teléfono [593] 032498288

\section{Conflicto de intereses: Los autores declaran no tener conflictos de intereses.}

Fondos: Ver la página 188

Recibido: 19 Enero 2017

Aceptado: 15 Noviembre 2018

Publicado: 30 Diciembre 2018

Membrete bibliográfico:

Gavilanes A, Martínez G. Cáncer de Mama, siete años de experiencia quirúrgica en el Hospital Oncológico "Julio Enrique Paredes C". Rev. Oncol. Ecu 2018;28(3):181-190.

DOI: $\underline{\text { https://doi.org/10.33821/205 }}$

Copyright Gavilanes, et al. Este artículo es distribuido bajo los términos de Creative Commons Attribution License, el cual permite el uso y redistribución citando la fuente y al autor original.

\section{Breast Cancer", Seven year's of surgilcal experience at the "Julio Enrique Paredes C"Oncology Hospital.}

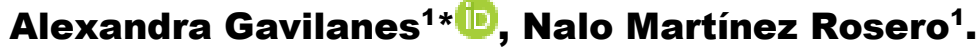

1. Hospital Oncológico "Julio Enrique Paredes C", Unidad oncológica SolcaTungurahua, Ecuador.

\section{Resumen}

Introducción: En la última década se han incorporado nuevas técnicas quirúrgicas como la cirugía oncoplástica conservadora y la biopsia de ganglio centinela para el tratamiento de cáncer de mama. El objetivo del presente estudio es describir el tipo de cirugía realizados en mujeres con cáncer de mama en un centro oncológico.

Métodos: El presente estudio descriptivo, retrospectivo, fue realizado en el Hospital Oncológico "Julio Enrique Paredes C" Solca-Ambato. Se revisaron las historias clínicas del período 2008 - 2014 de pacientes con cáncer de mama. Las variables analizadas fueron: edad, localización del tumor, estadío clínico y tipo de cirugía.

Resultados: En el hospital fueron sometidas a tratamiento quirúrgico 114 pacientes con cáncer de mama, entre 40 y 69 años; el carcinoma ductal infiltrante en un $69 \%$; el estadio clínico II corresponde a un $49 \%$; la mayor ubicación fue en el cuadrante superior externo de mama izquierda. Un 59 \% fue sometido a mastectomía radical modificada. 12 \% de casos se realizaron reconstrucción mamaria con colgajo del músculo recto transverso del abdomen.

Conclusión: En este reporte se un incrementando el número de casos de cáncer de mama, que deben ser sometidos a tratamiento quirúrgico, en los últimos años se ve una mayor incidencia de cirugías conservadoras de la mama.

Palabras Claves: NEOPLASIAS DE LA MAMA, MASTECTOMÍA SEGMENTARIA, MASTECTOMÍA.

DOI: $10.33821 / 205$ 


\section{Abstract}

Introduction: In the last decade new surgical techniques have been incorporated, such as conservative oncoplastic surgery and sentinel lymph node biopsy for the treatment of breast cancer. The objective of the present study is to describe the type of surgery performed in women with breast cancer in a cancer center.

Methods: The present descriptive, retrospective study was carried out in the Oncology Hospital "Julio Enrique Paredes C" Solca-Ambato. The clinical histories of the period 2008-2014 of patients with breast cancer were reviewed. The variables analyzed were: age, tumor location, clinical stage and type of surgery.

Results: 114 patients with breast cancer, between 40 and 69 years old, underwent surgical treatment at the hospital; infiltrating ductal carcinoma in $69 \%$; Clinical stage II corresponds to $49 \%$; the largest location was in the upper external quadrant of the left breast. $59 \%$ underwent a modified radical mastectomy. $12 \%$ of cases were performed breast reconstruction with transverse rectus abdominis muscle flap.

Conclusion: In this report there is an increase in the number of cases of breast cancer, which should be submitted to surgical treatment, in recent years we see a higher incidence of breast-conserving surgeries.

Keywords: BREAST NEOPLASMS, SEGMENTARY MASTECTOMY, MASTECTOMY.

DOI: $10.33821 / 205$

\section{Introducción}

Según la Organización Mundial de la Salud, en el mundo se presentan alrededor de 500.000 nuevos casos de cáncer por año y se producen alrededor de 250.000 muertes por esa causa. Del mismo modo, en Ecuador se presentan cerca de 10.200 nuevos casos por año y fallecen unas 4.000 mujeres [1].

El Registro Nacional de Tumores de Solca, de 2009, señala que 14 de cada 100 ecuatorianos murieron de cáncer [2]. De acuerdo con la OPS, Ecuador posee una de las tasas anuales más altas de cáncer en Latinoamérica, con el $11.6 \%$, mientras que el promedio de la región alcanza el $9 \%$ [3].

En los últimos años se han producido avances importantes en el diagnóstico y tratamiento de la patología mamaria, con gran impacto en el manejo de pacientes con Cáncer de Mama. Todo ello ha permitido el diagnóstico de tumores en estadio precoz.

El tratamiento quirúrgico persiste como un tratamiento imprescindible y en ocasiones el único para pacientes con cáncer de mama. En los últimos años se han incrementado nuevas opciones quirúrgicas, esto se debe al progreso en el diagnóstico y en los tratamientos no quirúrgicos lo que explica el aumento de la cirugía conservadora sobre la mastectomía. Otros cambios son debidos a la implantación de nuevas técnicas como la cirugía oncoplástica de mama o la biopsia de ganglio centinela. Esto ha favorecido a mejorar la supervivencia y calidad de vida de mujeres con Cáncer de Mama [4]. 
Un tratamiento quirúrgico correcto es imprescindible para conseguir la curación de las pacientes con cáncer de mama. El cirujano teniendo en cuenta, la edad del paciente, el tipo histopatológico, estadio clínico, ubicación y tamaño del tumor, debe elegir la mejor cirugía en la mama y ganglios axilares. En la última década se han incorporado nuevas técnicas quirúrgicas como la cirugía oncoplástica conservadora y la biopsia de ganglio centinela. La cirugía oncoplástica aporta seguridad oncológica y buen resultado estético [5].

El presente estudio tiene como objetivo describir el tipo de cirugía realizados en mujeres con cáncer de mama en un centro oncológico.

\section{Materiales y Métodos}

Estudio descriptivo, retrospectivo realizado en el Hospital Oncológico "Julio Enrique Paredes C", Solca - Tungurahua, a partir de las historias clínicas de los pacientes que cumplieron los criterios de inclusión, en los años 2008 y 2014.

El universo lo conforman todos los pacientes tratados con cirugía de cáncer de mama. Los datos de incidencia de cáncer proceden del registro de tumores de SOLCA Tungurahua. La fuente de información procede del registro de datos de la unidad oncológica donde se realizó el estudio. Para la fase de recolección se utilizó un formulario que consta de identificación del paciente, datos diagnósticos, y tratamiento quirúrgico al que se sometieron. El procesamiento de datos se realizó en el sistema informático del registro de tumores de SOLCA Tungurahua.

Las variables descritas fueron edad, localización del tumor, estadío clínico al cual fue intervenido, tipo de cirugía. Se reportan variables descriptivas. El estudio contó con aprobación de la Jefatura del Servicio y Dirección Médica para realizar este reporte.

\section{Resultados}

En el estudio se incluyeron 114 pacientes, el grupo de edad con mayor incidencia de cáncer de mama fue entre los 60 a 69 años, el segundo grupo de prevalencia es de 40 a 49 años (Figura 1). El mayor número de casos se presentó en los años 2013 y 2014.

La ubicación más frecuente (30 \%) fue en el cuadrante supero externo, el cuadrante supero interno $13 \%$ y la zona retroaerolar $12 \%$ y con mayor frecuencia en mama izquierda con 66 casos que corresponde al $66.58 \%$ (Figuras 2 y 3 ).

La histopatología más prevalente fue el carcinoma ductal infiltrante 78 casos (69\%), seguido del carcinoma micropapilar con 11 casos (9\%) y con menor incidencia los otros tipos de cáncer como son linfoma y sarcoma de mama que corresponde al 1\% (Figura 4). El mayor número de casos fueron diagnosticados en Estadío Clínico II (49\%), seguido del Estadío Clínico III (24 \%), Estadío Clínico I (23 \%), Estadío Clínico IV (4 \%). 
El mayor número de cirugías correspondió a Mastectomía Radical Modificada (59\%), el 14 $\%$ correspondiente a cuadrantectomía. Además se puede observar que durante los últimos años se ha incrementado el número de cirugías conservadoras y cirugías oncoplásticas con reconstrucción mamaria tipo TRAM correspondiendo al 12 \% número de casos en el 2014 como se puede observar en las Figuras 5 y 6.

Figura 1. Edad del grupo de estudio de acuerdo al año de diagnóstico en pacientes sometidos a tratamiento quirúrgico en el Hospital Oncológico "Julio Enrique Paredes C" período 2008 al 2014.

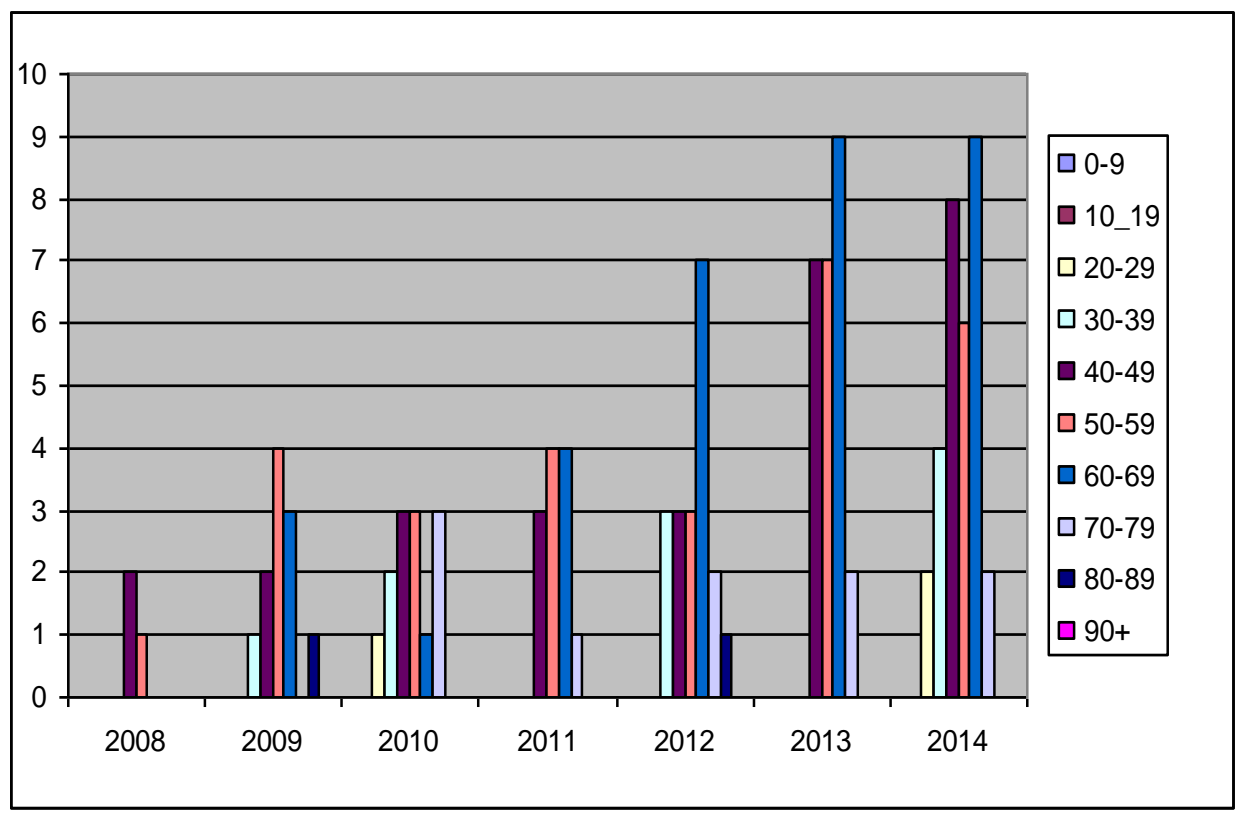

Figura 2. Distribución de pacientes con cáncer de mama según la localización del tumor en pacientes sometidos a tratamiento quirúrgico en el Hospital Oncológico "Julio Enrique Paredes C" En el periodo 2008-2014.

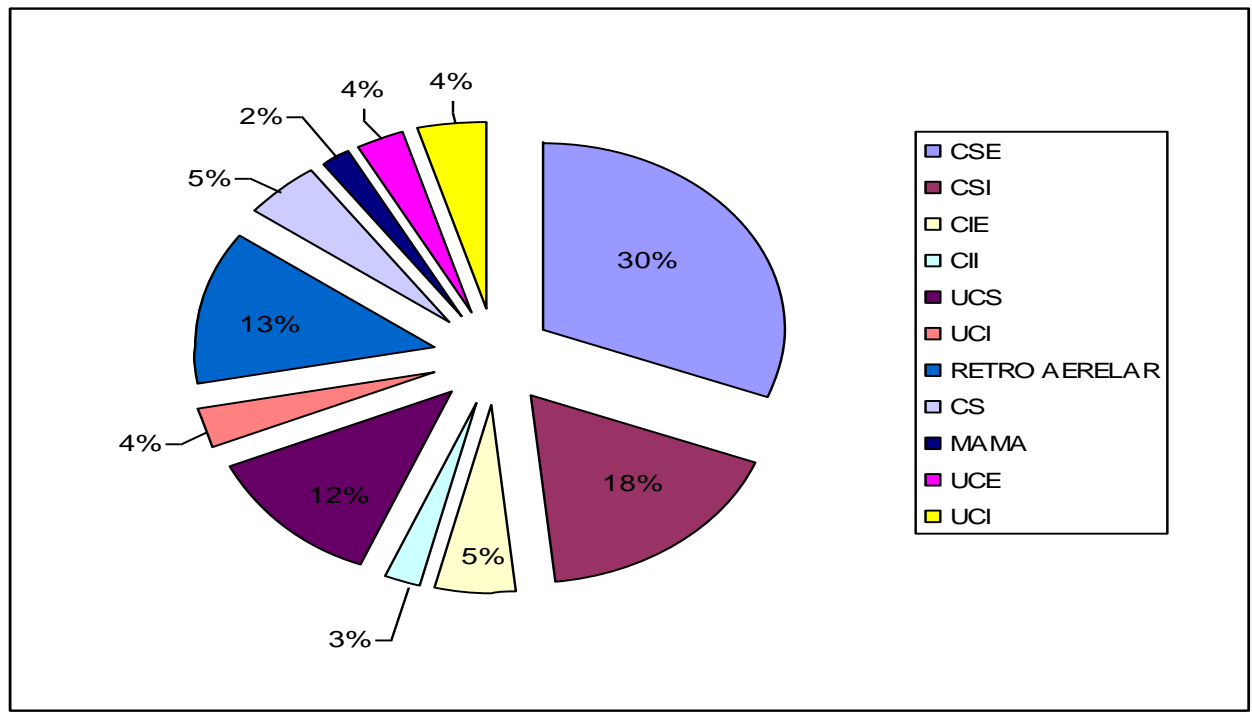


Figura 3. Distribución de pacientes con cáncer de mama según la localización del tumor en pacientes sometidos a tratamiento quirúrgico en el Hospital Oncológico "Julio Enrique Paredes C" En el periodo 2008-2014.

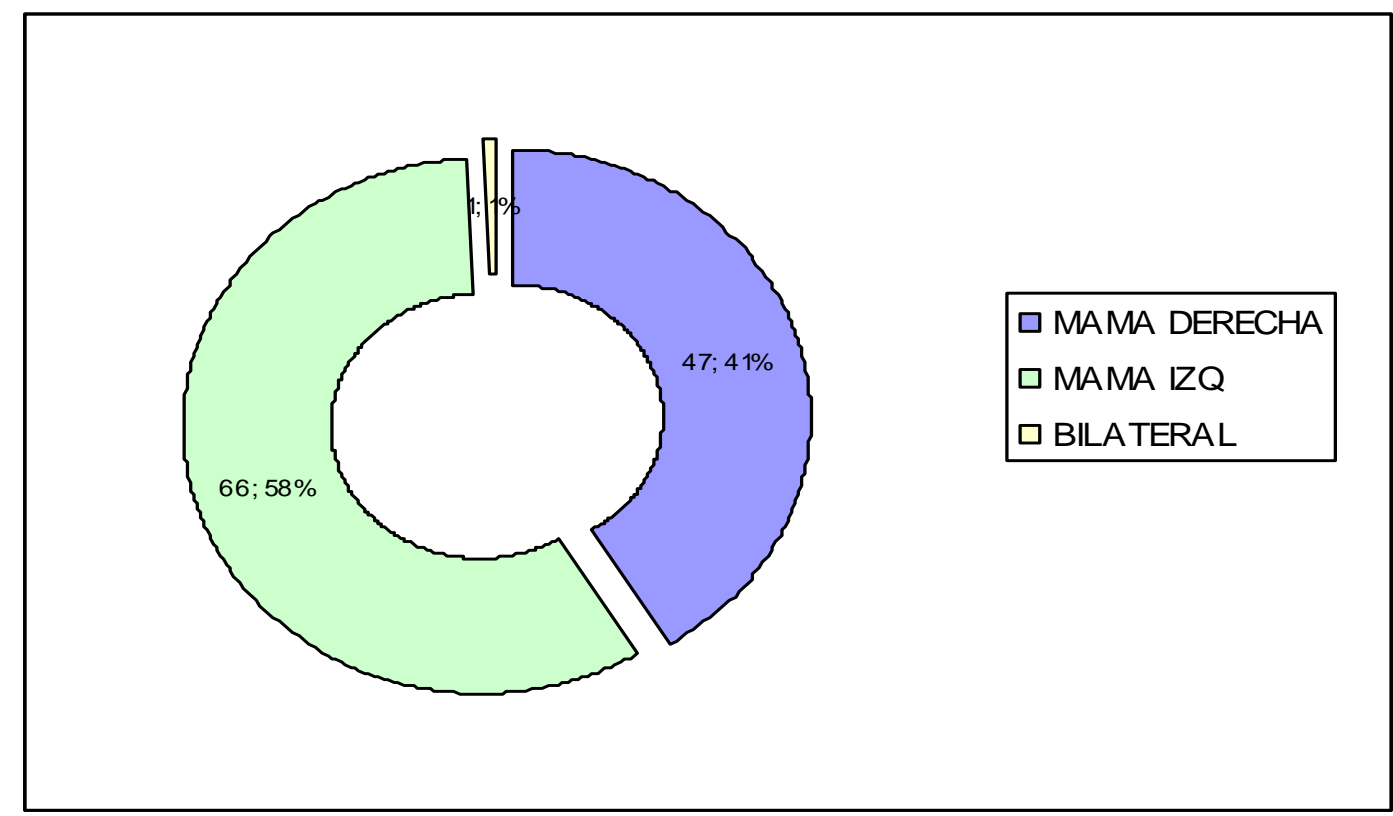

Figura 4. Morfología del Ca de mama según el estudio histopatológico desde el 2008 -2014 en pacientes sometidos a tratamiento quirúrgico en el Hospital Oncológico "Julio Enrique Paredes C"

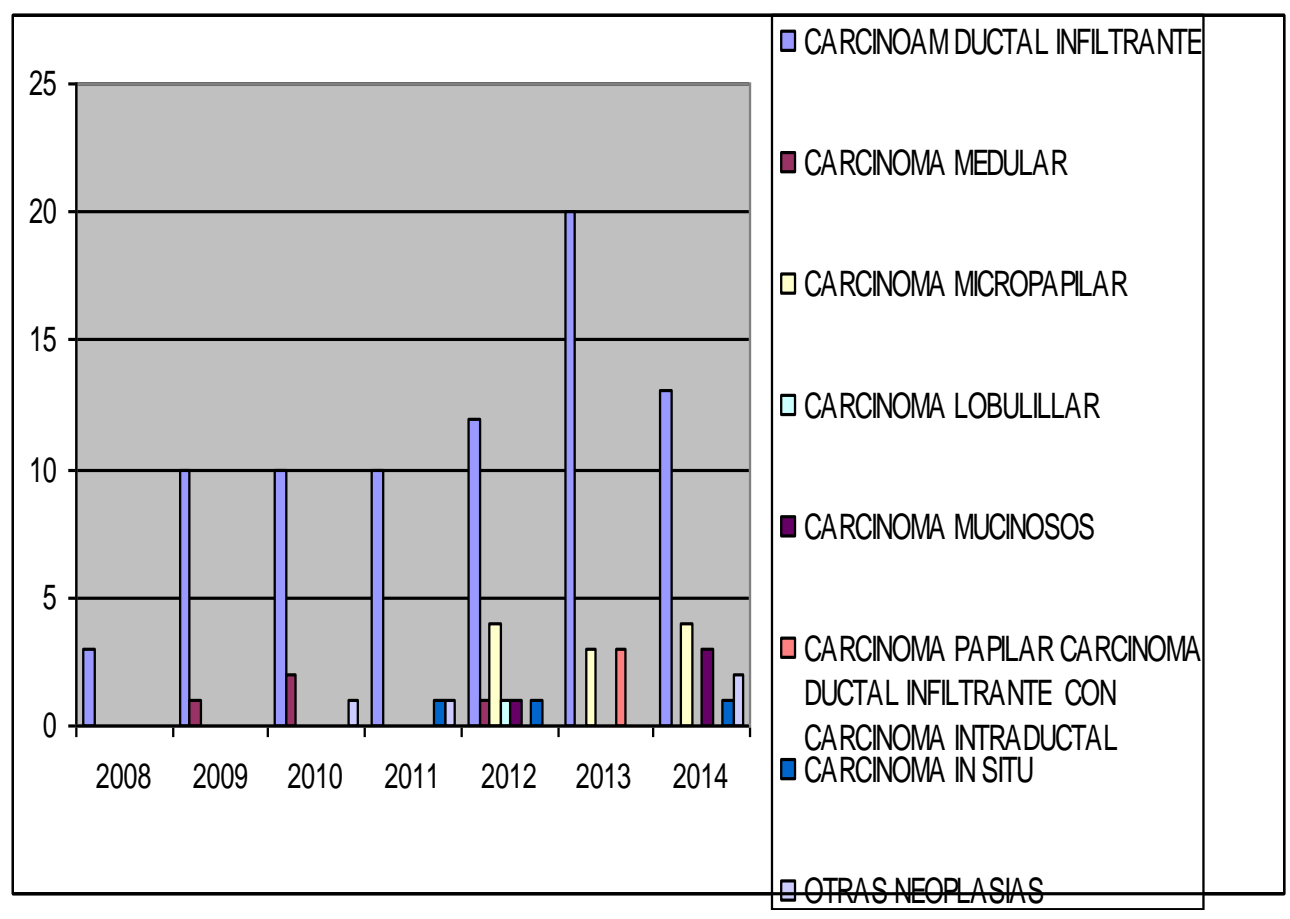


Figura 5. Tipos de cirugía realizadas en el Hospital Oncológico "Julio Enrique Paredes C" desde el año 2008 al 2014.

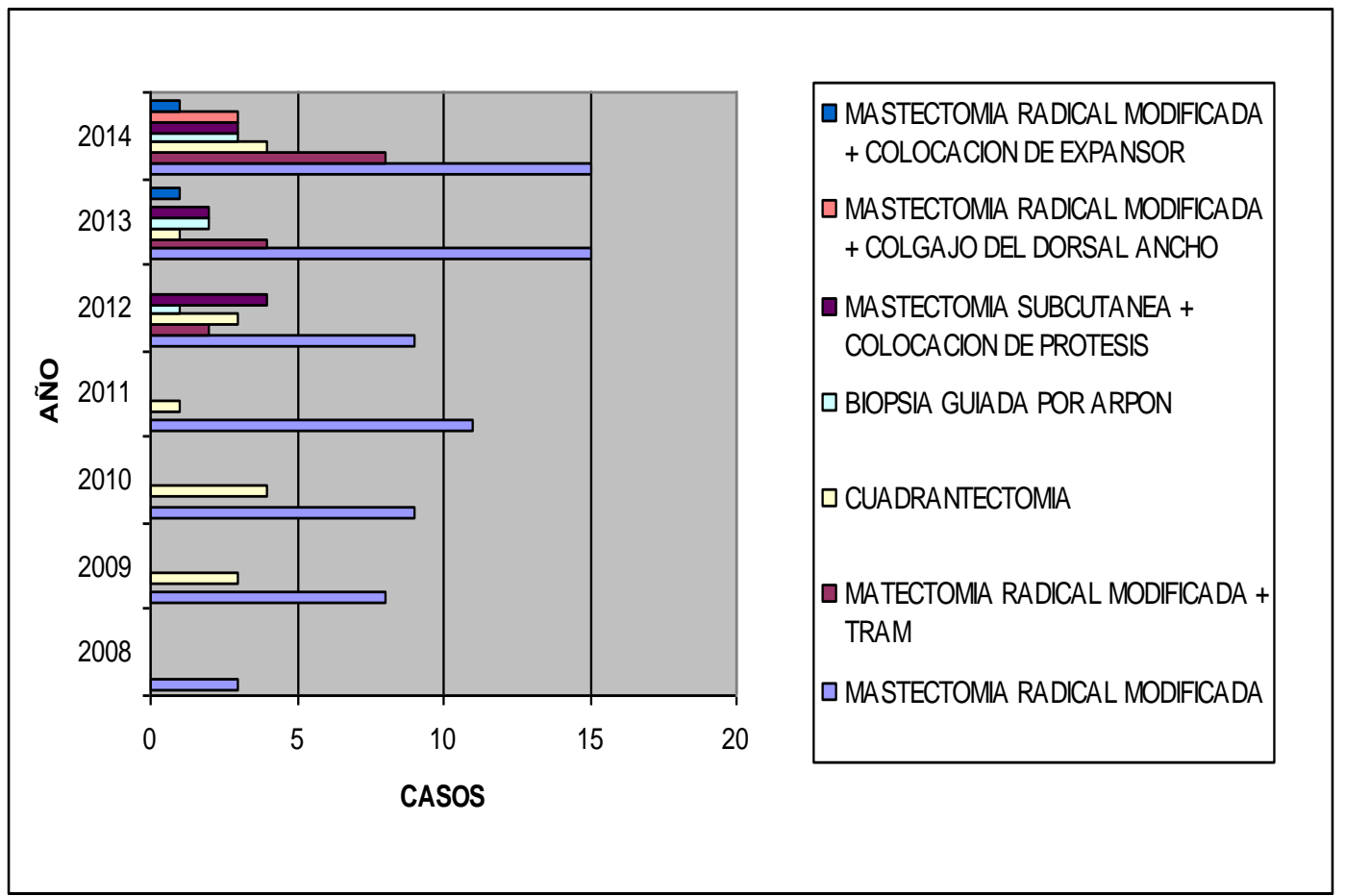

Figura 6. Tipos de cirugía realizadas en el Hospital Oncológico "Julio Enrique Paredes C" desde el año 2008 al 2014.

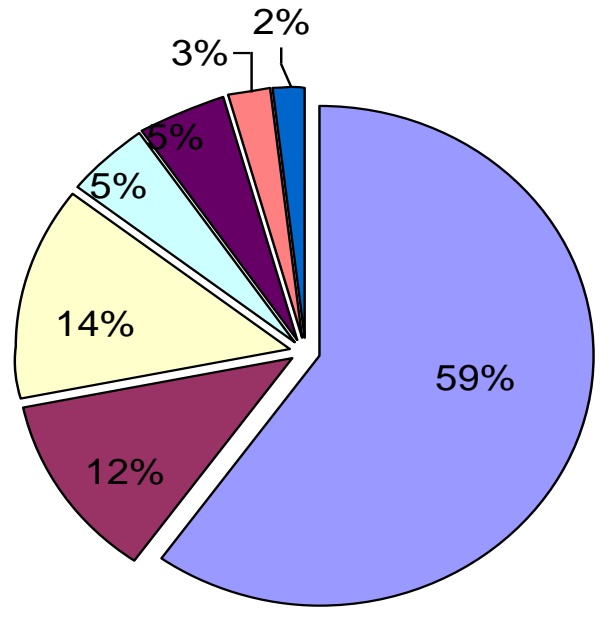

$\square$ MASTECTOMIA RADICAL MODIFICADA

口 MATECTOMIA RADICAL MODIFICADA + TRAM

$\square$ CUADRANTECTOMIA

$\square$ BIOPSIA GUIADA POR ARPON

- MASTECTOMIA SUBCUTANEA + COLOCACION DE PROTESIS

$\square$ MASTECTOMIA RADICAL MODIFICADA + COLGAJO DEL DORSAL ANCHO

- MASTECTOMIA RADICAL MODIFICADA + COLOCACION DE EXPANSOR 


\section{Discusión}

Se describe en el presente reporte que la edad de grupo de estudio de mujeres con cáncer de mama fue entre los 60 a 69 años, el segundo grupo de prevalencia es de 40 a 49 años lo que coincida con reportes internacionales. Este estudio describe que la ubicación del cáncer de mama más frecuente es cuadrante supero externo lo que es de común reporte en estudios [6-10].

La histopatología más prevalente fue el carcinoma ductal infiltrante y el mayor número de casos fueron diagnosticados en Estadío Clínico II (49\%), lo que induce a pensar que los diagnósticos de cáncer de mama se están realizando en estadios clínicos más tempranos lo que permite ofrecer al paciente un tratamiento adecuado como la quimioterapia adyuvante previo al tratamiento quirúrgico que ofrece un mayor margen de seguridad en el procedimiento, solo un $4 \%$ fue sometido a tratamiento quirúrgico en EC IV como método de limpieza en tumores fungados o con una gran carga tumoral.

El mayor número de cirugías correspondió a Mastectomía Radical Modificada (59 \%), sin embargo el reporte presenta que durante los últimos años que se realizaron cirugías oncoplásticas con reconstrucción mamaria con colgajo del músculo recto transverso del abdomen (tipo TRAM) lo que indica que el tipo de cirugías conservadoras del cáncer de mama empezaría a realizarse en los años 2013 y 2014 . Ha habido una evolución continua de la extirpación radical de los siglos 19 y 20, que implica la pérdida total de la mama, la piel y el músculo subyacente.

Los procedimientos individualizados actuales tienen como objetivo la extirpación completa del tumor preservando tanto como sea posible la estructura de la mama. Con el paso de los años la experiencia quirúrgica aumenta en el equipo médico lo que hace posible el aumentando las cirugías conservadoras y oncoplástica, lo que permite mejorar autoestima de las pacientes sometidas a cirugía y por ende su calidad de vida. La evaluación de la biopsia del ganglio centinela en la estadificación ha reducido las intervenciones axilares anteriormente extensas a niveles mínimamente invasivos; con la posibilidad de hacer aún menos en la axila, datos no presentados en este informe.

La cirugía ha sido durante mucho tiempo la piedra angular del tratamiento del cáncer, el enfoque más directo de la extinción de un tumor. El campo ha hecho avances notables en los últimos 50 años y sigue avanzando a una velocidad vertiginosa como las nuevas técnicas permiten a los cirujanos realizar operaciones que antes se consideraban imposibles.

El cambio más grande será el uso de la imagen molecular intraoperatoria, método en el cual se encienden las células cancerosas para poder ver con claridad y garantizar la eliminación de todas [durante la cirugía].

Ya hemos visto notables avances en la tecnología de imágenes, que han mejorado nuestra capacidad de visualizar un tumor. Ahora podemos vincular un trazador radioactivo a los anticuerpos que se unen a las células cancerosas y ver el cáncer con un PET scan mucho mejor que con los métodos de imagen estándar. 


\section{Conclusiones}

En este reporte se un incrementando el número de casos de cáncer de mama, que deben ser sometidos a tratamiento quirúrgico, en los últimos años se ve una mayor incidencia de cirugías conservadoras de la mama.

\section{Agradecimientos}

Reconocemos a las personas que participaron indirectamente en el estudio tales como el personal técnico y otras en general del Hospital Oncológico "Julio Enrique Paredes C", Unidad oncológica Solca-Tungurahua, Ecuador, a quienes agradecemos.

\section{Información adicional}

Nota del Editor

La Revista Oncología Ecu permanece neutral con respecto a los reclamos jurisdiccionales en mapas publicados y afiliaciones institucionales.

\section{Abreviaturas}

TRAM: Siglas en Inglés del músculo recto transverso del abdomen.

OPS: Organización Panamericana de la salud.

\section{Archivos Adicionales}

Ninguno declarado por los autores.

\section{Fondos}

Los fondos de la investigación fueron propios de los autores del presente artículo.

\section{Disponibilidad de datos y materiales}

Existe la disponibilidad de datos bajo solicitud al autor de correspondencia. No se reportan otros materiales. 
AG, NM, realizaron la idea de investigación, revisión bibliográfica, recolección de datos, escritura del artículo. NM realizó el análisis crítico del artículo. Todos los autores leyeron y aprobaron la versión final del artículo.

Aprobación de ética y consentimiento para participar

No aplica ya que es un estudio retrospectivo

\section{Consentimiento para publicación}

No aplica.

\section{Información de los autores}

Alexandra Gavilanes, Médico General, Residente 2 de la Unidad Oncológica SolcaTungurahua (D) https://orcid.org/0000-0003-4361-7433

Nalo Martínez, Director Médico de la Unidad Oncológica Solca Tungurahua.

Abreviaturas en la referencias

DOI: Digital Object

Identifier

PMID: PubMed Identifier

SU: Short URL
Revisiones por pares

Acceda a la revisión de pares académicos en el siguiente enlace: https://publons.com/review/3734958/

\section{Referencias}

1. Regueira F, Rodríguez N, García M, Zornoza G. Novedades en el tratamiento quirúrgico del cáncer de mama. Rev Med Univ Navarra 2008;52 (1):51-55. http://hdl.handle.net/10171/35398

2. Sociedad de Lucha Contra el Cancer-Solca. Registro de Tumores SOLCA TUNGURAHUA 2009. Reporte Institucional.

3. Scardino P. Breast cancer. Memorial Sloan Kettering Cancer Center. NY. 2015

4. Memorial Sloan Kettering Cancer Center, NY. Mskcc.org. Breast Cancer. 2015. https://www.mskcc.org/cancer-care/types/breast/types-breast

5. Breasted J. The Edwin Smith surgical papyrus. Special ed. Birmingham, Ala: Classics of Medicine Library; 1984.

6. Halsted W. The results of operations for the cure of cancer of the breast performed at the Johns Hopkins Hospital from june, 1889, to january, 1894. Annals of Surgery. 1894;20(5):4-497. DOl: 10.1097/00000658-189407000-00075

7. Halsted W. The results of radical operations for the cure of carcinoma of the breast. Annals of Surgery. 1907;46(1):1-19. DOI: $10.1097 / 00000658-190707000-00001$ 
8. Edge S, Byrd D, Compton C, Greene F, Fritz A, Tampa A. AJCC Cancer Staging Manual. JAMA. 2010;304(7):347-7 DOI: 10.1001/jama.2010.1525

9. Le Dran H. Memoires avec un précis de plusieures observation sur le cáncer. Mémoires de l'Académie royale de chirurgie.3.1 ed. Paris: Chez P. Al. Le Prieur; 1757.

10. Cotlar A, Dubose J, Rose D. History of surgery for breast cancer: radical to the sublime. Current Surgery. 2003;60(3):329-33. DOI: 10.1016/S0149-7944(02)00777-8 